\title{
AVALIAÇÃO DA IMPLEMENTAÇÃO DE COMISSÃO MULTIDISCIPLINAR PARA O CUIDADO DE IDOSOS COM FRATURA DE FÊMUR PROXIMAL
}

\author{
Amanda Patissi Giacomelli Leal ${ }^{a}$ \\ Cristina Terumi Okamoto ${ }^{b}$ \\ Gabrielle Bornancin Costa ${ }^{\mathrm{C}}$ \\ Hanne Lise Chaves ${ }^{d}$ \\ José Mauro Regula Esposito e \\ Maria Julia Macedo Bonatto $f$ \\ Mateus José Schmitz ${ }^{9}$
}

abcdefg Departamento de Medicina, Universidade Positivo. R. Prof. Pedro Viriato Parigot de Souza, 5300 - Cidade Industrial De Curitiba, Curitiba - PR, 81280-330.

\section{RESUMO}

Introdução: A fratura de fêmur proximal é um dos traumas mais frequentes em idosos e está relacionada a fatores como osteoporose, sarcopenia e fragilidade senil. Suas consequências podem ser desastrosas, acarretando declínio da capacidade funcional, perda de autonomia e redução da expectativa de vida desses pacientes. Objetivo: comparar o tempo de internamento, tempo até o tratamento da fratura e mortalidade em idosos com fratura de fêmur proximal antes e após a implantação de uma comissão multidisciplinar. Material e Métodos: trata-se de um estudo transversal com coleta de dados retrospectivos através da análise de prontuários de pacientes internados em um hospital de trauma em Curitiba/PR., no período de outubro de 2015 a fevereiro de 2016 e de abril a agosto de 2016. Foram incluídos pacientes com idade igual ou superior a 60 anos, internados no hospital com diagnóstico de fratura de fêmur proximal, através dos códigos do CID-10 S72.0, S72.1 e S72.2. Resultados: o tempo de internamento e o tempo entre o diagnóstico da fratura e seu tratamento foram significativamente menores após a implantação da comissão multidisciplinar ( $p=0,002$ e redução mediana de 3 dias; $p<0,001$ e redução mediana de 2,5 dias, respectivamente); houve uma tendência estatística à redução da taxa de óbitos após a criação da comissão $(15,9 \%$ vs. $7 \% ; p=0,065)$. Conclusão: observou-se que idosos com fratura de fêmur proximal se beneficiam de um atendimento multidisciplinar devido à redução do tempo de internamento e menor espera até o tratamento definitivo, com tendência à redução da taxa de mortalidade intra-hospitalar.

Palavras-chave: Idoso; Fraturas do quadril; Tempo de internação.

*Autor correspondente: Amanda Patissi Giacomelli Leal, Graduanda em Medicina, R. Coronel Joaquim Ignácio Taborda Ribas, 750 - Ctba/PR. Telefone de contato: (41) 99700-1880. E-mail de contato:

amandapgiacomelli@gmail.com. 


\section{INTRODUÇÃO}

A fratura de fêmur proximal está entre as lesões traumáticas mais frequentes nos idosos $^{(1)}$. Esta lesão é particularmente devastadora para a saúde e capacidade funcional desses pacientes, com somente $25 \%$ deles retornando às suas atividades habituais, $40 \%$ tornando-se dependentes de terceiros e $20 \%$ evoluindo a óbito dentro de um ano após a lesão(2-4). Além disso, é estimada uma redução de 15 a $20 \%$ na expectativa de vida desses indivíduos, com um risco relativo de morte aumentado em 4\% ao ano ${ }^{(5,6)}$.

Esse tipo de fratura se relaciona a fatores como osteoporose, sarcopenia e diminuição das atividades de vida diária, sendo mais comum em idosos frágeis ${ }^{(1,7)}$. Dessa forma, o risco para fratura de fêmur proximal dobra a cada década após os 50 anos de idade, e sua incidência aumenta a cada ano, acompanhando o aumento da prevalência de idosos na população, avançando como um grave problema de saúde pública ${ }^{(5,8)}$. Cerca de $86 \%$ dos casos ocorrem em pessoas com mais de 65 anos e a maioria desses pacientes apresenta comorbidades pré-existentes ou incapacidades físicas, exigindo um tratamento integral e complexo ${ }^{(9)}$.

Não obstante, cerca de $48 \%$ dos idosos com fratura de quadril apresentam de duas a seis comorbidades associadas, número diretamente proporcional ao período de internamento(10). Assim sendo, o cuidado hospitalar para este tipo de fratura se torna complexo e desafiador além de gerar custos elevados para o sistema. Nos Estados Unidos, por exemplo, os gastos com fratura de quadril chegam a um terço dos gastos com fraturas em geral(11). A implantação de unidades de cuidados multidisciplinares mostra-se uma alternativa para fornecer tratamento adequado a esses pacientes, objetivando prevenir complicações intra-hospitalares e melhorar a recuperação funcional, com rapidez de tratamento, melhores resultados e menores $\operatorname{custos}^{(12,13) .}$

Ciente desses dados, em março de 2016 o Hospital do Trabalhador, localizado em Curitiba (PR/Brasil) implantou a Comissão
Multidisciplinar de Acompanhamento das Fraturas Agudas (COMAFA), composta por médicos, enfermeiros, fisioterapeutas, nutricionistas, psicólogos e assistentes sociais, com o objetivo de otimizar o tratamento clínico global dos idosos. Este hospital é referência no atendimento de pacientes ortopédicos e politraumatizados na cidade, e entre aqueles com fratura de fêmur proximal, grande parcela constitui-se de idosos.

Após a criação da COMAFA foram desenvolvidos protocolos internos no hospital com a finalidade de articular os setores envolvidos no cuidado do idoso com fratura de fêmur. Nesse sentido, o pronto atendimento, o corpo clínico, o corpo cirúrgico, a Unidade de Terapia Intensiva (UTI), os serviços multidisciplinares (fisioterapia, nutrição, psicologia e assistência social) e os laboratórios estão preparados para receber e tratar esses pacientes, com acionamento simultâneo do corpo clínico e da ortopedia, reserva precoce de vaga em UTI e prioridade de resultados laboratoriais desses pacientes assim que realizado o diagnóstico da fratura. Além disso, reuniões semanais são realizadas para discussão multidisciplinar dos casos encaminhados à comissão, para a elaboração de um plano terapêutico que atenda as demandas particulares dessa população. O objetivo é que o tratamento cirúrgico da fratura seja realizado de preferência dentro de $72 \mathrm{~h}$ da admissão, na tentativa de minimizar o tempo de internamento e melhorar a recuperação desses idosos.

A implementação da COMAFA é recente, sendo indispensável a análise de seu impacto no atendimento de idosos, principalmente quanto à diminuição do tempo de internação e diminuição das complicações intrahospitalares.

Dessa forma, o objetivo de nosso estudo é verificar o impacto de uma equipe multidisciplinar no internamento de idosos com fratura de fêmur proximal, comparando o período de permanência hospitalar, o tempo decorrido entre o diagnóstico e o tratamento definitivo da fratura, o período de internamento em UTI, taxas de infecção intra-hospitalar, mortalidade e necessidade de reinternação 
antes e após a implementação da comissão multidisciplinar.

\section{MATERIAL E MÉTODOS}

\subsection{Tipo De Estudo}

Estudo transversal, com coleta retrospectiva de dados.

\subsection{População}

Foram analisados os prontuários de pacientes com idade igual ou superior a 60 anos.

\subsection{Local}

O estudo foi desenvolvido no Complexo Hospitalar do Trabalhador, localizado em Curitiba/PR, um hospital de ensino que é referência nas áreas de trauma e emergência, materno-infantil, saúde do trabalhador e infectologia.

\section{4 Critérios De Seleção}

Estiveram aptos a participar do estudo os prontuários de pacientes com idade igual ou superior a 60 anos, internados nos períodos de outubro de 2015 a fevereiro de 2016 (grupo 1, anterior à implantação da COMAFA) e de abril de 2016 a agosto de 2016 (grupo 2, posterior à implantação da COMAFA), com diagnóstico de fratura de fêmur proximal, registrados com os códigos da Classificação Internacional de Doenças, versão 10 (CID-10) S72.0, S72.1 e S72.2. Foram excluídos os idosos politraumatizados, com fratura de fêmur bilateral e com fratura patológica decorrente de tumores ou infecções prévias.

Pela alta prevalência de osteoporose na população estudada, fraturas em pacientes com essa moléstia em conjunto com mecanismo de trauma de intensidade que justifique uma fratura em indivíduos hígidos não foram consideradas como patológicas, sendo incluídas no estudo.

\subsection{Coleta de Dados}

A coleta de dados foi realizada nos meses de novembro a dezembro de 2019. Os dados foram coletados do prontuário eletrônico dos pacientes com auxílio de um questionário previamente elaborado pelos autores. Os prontuários eletrônicos foram identificados por meio dos seus respectivos números de registro, sem coleta ou exposição dos nomes dos pacientes.

Os dados coletados foram: idade, sexo, código do CID-10 para diagnóstico da fratura (S72.0, S72.1 e S72.2), tempo de internamento total e em UTI, período decorrido entre o diagnóstico e o tratamento da fratura, ocorrência de infecção hospitalar e sítio infeccioso, desfecho do caso (óbito ou alta) e necessidade de reinternação após a alta hospitalar.

Para determinar a ocorrência de infecção hospitalar utilizou-se a definição da portaria $n^{\circ}$ 2616/1998 do Ministério da Saúde que expede as diretrizes e normas para prevenção e controle de infecções hospitalares. Portanto, considerou-se como infecção hospitalar o processo infeccioso adquirido pelo paciente a partir de $72 \mathrm{~h}$ após sua admissão hospitalar, ou antes desse período, quando associado a um procedimento diagnóstico e/ou terapêutico realizado nesse intervalo de tempo e descrito em prontuário.

Considerou-se como reinternação a readmissão hospitalar até 30 dias após a alta por motivo ligado ao internamento anterior.

\subsection{Análise e tratamento dos dados}

A organização dos dados foi feita através da construção de banco de dados por meio do programa Microsoft Excel 2016. Os dados foram tabulados e analisados com os pacotes estatísticos GRAPHPAD PRISM. As associações entre as variáveis estudadas foram avaliadas segundo testes exatos de Fisher; as diferenças entre médias foram testadas através do teste Mann-Whitney, uma vez que não foi comprovada normalidade segundo o teste de D'Agostino \& Pearson. Os 
dados foram corrigidos por análise de regressão logística binária através do programa STATA v.9.2 (StataCorp, EUA). Na análise estatística, foi considerado um nível de significância $\alpha=0,05^{(14,15)}$.

\subsection{Aspectos Éticos}

Considerando os preceitos éticos, o estudo foi aprovado pelo Comitê de Ética em pesquisa do Hospital do Trabalhador/Secretaria de Estado da Saúde do Paraná sob o parecer $\mathrm{n}^{\circ}$ 3.602.157 em 26 de setembro de 2019 e atendeu a Resolução 466/2012, do Conselho Nacional de Saúde, que dispõe sobre pesquisas que envolvem seres humanos. Devido à pesquisa não envolver seres humanos diretamente e não possibilitar sua identificação, constituindo-se apenas da análise de prontuários sem exposição nominal, não foi necessário o Termo de Consentimento Livre e Esclarecido (TCLE), havendo sido assinada e aprovada a Dispensa do TCLE pelo Comitê de Ética referido.
Os autores declaram que não há conflitos de interesses factuais ou potenciais, sejam eles de natureza financeira, pessoal, acadêmica, relacionado à afiliação institucional, política e/ou religiosa em relação ao artigo.

\section{RESULTADOS E DISCUSSÃO}

O universo amostral do período analisado foi de 88 para o grupo 1 (controle) e de 100 para o grupo 2 (pós-COMAFA). A análise do perfil epidemiológico da amostra (Tabela 1) não mostrou diferença significativa entre os grupos em relação ao sexo $(p=0,1111)$ e à idade $(p=0,473)$, mesmo após análise de regressão logística binária (OR 2,04 95\%IC [0,99-4,19]) (Tabela 2). O sexo feminino predominou em ambos os grupos, representando $76,1 \%$ e $65 \%$ da população estudada; a maioria dos pacientes ficou na faixa etária de 81 a 90 anos nos dois períodos analisados (Tabela 1), com mediana de idade semelhante entre os grupos (Tabela 3).

Tabela 1 - Perfil epidemiológico da amostra, Curitiba/PR, Brasil, 2020

\begin{tabular}{lccc}
\hline Sexo & Pré COMAFA & Pós COMAFA & \\
$\mathrm{n}=88(100 \%)$ & $\mathrm{n}=100(100 \%)$ & $\mathbf{p}^{*}$ \\
\hline Feminino & $76,1 \%(\mathrm{n}=67)$ & $65 \%(\mathrm{n}=65)$ & 0,1111 \\
Masculino & $23,9 \%(\mathrm{n}=21)$ & $35 \%(\mathrm{n}=35)$ &
\end{tabular}

\section{Faixa etária}

\begin{tabular}{lll}
\hline $60-70$ anos & $17(19,3 \%)$ & $26(26 \%)$ \\
$71-80$ anos & $31(35,2 \%)$ & $24(24 \%)$ \\
$81-90$ anos & $36(41,0 \%)$ & $38(38 \%)$ \\
$91-100$ anos & $4(4,500 \%)$ & $11(11 \%)$ \\
Mais de 100 anos & $0(0,000 \%)$ & $1(1,0 \%)$ \\
\hline
\end{tabular}

Fonte: autores, 2020

*Variáveis analisadas através do teste exato de Fisher. Foi considerado como estatisticamente significante valores de $p<0,05$. 
Tabela 2 - Regressão Logística Binária pré COMAFA vs. pós COMAFA, Curitiba/PR, Brasil, 2020

\begin{tabular}{lccc}
\hline Pré vs. Pós $\quad \mathbf{n = 1 8 4}$ & $\mathbf{O R}$ & $\mathbf{9 5 \%} \mathbf{~ I C}$ & $\mathbf{p}^{*}$ \\
\hline Tempo da fratura até tratamento & 0,83 & $0,76-0,91$ & 0,000 \\
Necessidade de reinternação & 0,32 & $0,11-0,84$ & 0,021 \\
Sexo & 2,04 & $0,99-4,19$ & 0,052 \\
Comorbidades & 0,63 & $0,44-0,88$ & 0,008 \\
\hline
\end{tabular}

Fonte: os autores, 2020

n: número de observações incluídas

*Variáveis analisadas através do teste exato de Fisher. Foi considerado como estatisticamente significante valores de $p<0,05$.

Tabela 3 - Mediana das variáveis pré e pós COMAFA, Curitiba/PR, Brasil, 2020

\begin{tabular}{lccc}
\hline Variável & $\begin{array}{c}\text { Pré COMAFA } \\
(\mathbf{n = 8 8})\end{array}$ & $\begin{array}{c}\text { Pós COMAFA } \\
(\mathbf{n = 1 0 0})\end{array}$ & "p \\
\hline Idade & $79,5[72-85]$ & $80,5[70-88]$ & 0,473 \\
Tempo de internamento (dias) & $12[8-17]$ & $9[7-13]$ & 0,002 \\
Tempo de internamento em UTI (dias) & $3[1-6,7]$ & $3[1-5]$ & 0,223 \\
Tempo da fratura até tratamento (dias) & $5,5[3-8,7]$ & $3[1-5]$ & $<0,001$ \\
& & & \\
\hline
\end{tabular}

Fonte: autores, 2020

Nota: variáveis sem distribuição normal. Valores expressos em mediana [intervalo interquartil]

*Variáveis analisadas através do teste exato de Fisher. Foi considerado como estatisticamente significante valores de $p<0,05$.

O predomínio do sexo feminino em nosso estudo está de acordo com o descrito na literatura, onde é amplamente aceito que mulheres idosas são as principais vítimas de fratura de fêmur proximal $(16,17)$. Essa conformidade ocorre também em relação a idade média que na literatura varia de 78 a 82 $\operatorname{anos}^{(4,18,19) \text {. }}$

O tempo de internamento hospitalar apresentou redução estatisticamente significante com a implantação da COMAFA $(p=0,002)$, com uma mediana de 3 dias a menos (Tabela 3). Houve uma tendência semelhante em outros serviços que contavam com um grupo de interconsulta similar à COMAFA, com média de tempo de internamento de 11,7 dias $^{(4,20-23)}$. Alguns estudos que avaliaram a implantação de serviços multidisciplinares para o cuidado de idosos com fratura de quadril também demonstraram redução significativa no tempo de internamento dos pacientes após serem atendidos pela equipe ${ }^{(8,23)}$.

A análise descritiva dos tempos analisados mostrou predomínio de pacientes com 8 a 14 dias de internamento em ambos os períodos (Tabela 4). Não houve diferença estatisticamente significante no tempo de UTI $(p=0,223)$ entre os períodos analisados, com mediana de 3 dias de internamento em ambos (Tabela 3). Outros estudos descrevem tendência semelhante com média de tempo de UTI de cerca de 3 dias ${ }^{(21,22) . ~ A p e s a r ~ d e ~ n a ̃ o ~}$ haver redução do tempo médio de internamento em UTI os pacientes que pertencem ao grupo atendido pelas equipes multidisciplinares utilizam menos esse tipo de leito, o que pode contribuir inclusive para redução de $\operatorname{custos}^{(8,9)}$. Vale ressaltar que em nosso estudo os pacientes eram internados na UTI após a cirurgia como parte do protocolo assistencial, com objetivo de monitorar sua 
resposta ao tratamento, compensar suas comorbidades e prontamente agir em caso de complicações, o que pode ter sido um viés na avaliação do tempo de permanência em UTI.

O tempo decorrido entre o diagnóstico da

Tabela 4 - Descrição dos intervalos de tempo pré e pós COMAFA, Curitiba/PR, Brasil, 2020

\begin{tabular}{lcc}
\hline Tempo entre diagnóstico e tratamento & $\begin{array}{c}\text { Pré COMAFA } \\
\mathrm{n}=88(100 \%)\end{array}$ & $\begin{array}{c}\text { Pós COMAFA } \\
\mathrm{n}=100(100 \%)\end{array}$ \\
\hline $0-3$ dias & $25(28,5 \%)$ & $52(52 \%)$ \\
$4-6$ dias & $30(34 \%)$ & $34(34 \%)$ \\
$7-9$ dias & $13(14,8 \%)$ & $8(8 \%)$ \\
$10-12$ dias & $14(15,9 \%)$ & $4(4 \%)$ \\
Mais de 12 dias & $6(6,8 \%)$ & $2(2 \%)$ \\
& & \\
Tempo de internamento total & $\mathrm{n}=88(100 \%)$ & $\mathrm{n}=100(100 \%)$ \\
\hline $1-7$ dias & $16(18,2 \%)$ & $28(28 \%)$ \\
$8-14$ dias & $41(46,6 \%)$ & $55(55 \%)$ \\
$15-21$ dias & $16(18,2 \%)$ & $10(10 \%)$ \\
$22-28$ dias & $8(9,1 \%)$ & $3(3 \%)$ \\
Mais de 28 dias & $7(7,9 \%)$ & $4(4 \%)$ \\
& & \\
Tempo de internamento em UTI & $\mathrm{n}=88(100 \%)$ & $100(100 \%)$ \\
\hline $0-4$ dias & $58(66 \%)$ & $73(73 \%)$ \\
$5-9$ dias & $14(16 \%)$ & $20(20 \%)$ \\
$10-20$ dias & $9(10,1 \%)$ & $3(3 \%)$ \\
$21-30$ dias & $3(3,4 \%)$ & $3(3 \%)$ \\
Mais de 40 dias & $3(3,4 \%)$ & $0(0 \%)$ \\
\hline
\end{tabular}

Fonte: os autores, 2020

fratura e seu tratamento definitivo foi significativamente menor $(p<0,001)$ após a implantação da COMAFA, com redução mediana de 2,5 dias (Tabela 3). Após análise de regressão logística binária entre os períodos, o tempo do diagnóstico até 0 tratamento da fratura permaneceu estatisticamente menor (OR 0,83 95\% IC [0,76$0,91]$ ). No grupo 1 a maioria dos pacientes, 34 $\%$, foi tratada entre 4 a 6 dias após o diagnóstico enquanto no grupo 2 a maioria, $52 \%$, dentro de 0 a 3 dias (Tabela 4).

$\mathrm{Na}$ literatura os dados giram ao redor de uma média de 5,8 dias, sendo $13 \%$ dos pacientes tratados dentro de $24 \mathrm{~h}$ da ocorrência da fratura e quase $70 \%$ deles após $48 \mathrm{~h}$ da lesão. Apesar de muitos dos pacientes terem esperado mais de 2 dias para o tratamento definitivo não houve maior taxa de mortalidade $(p>0,05)^{(7)}$. Entretanto uma meta-análise demonstrou que idosos operados para fratura de quadril dentro de 1 a 2 dias após a admissão hospitalar tiveram redução significativa na mortalidade quando comparados àqueles tratados após o segundo dia (OR 0,74 95\%IC [0,67-0,81]; $p<0,000)^{(20)}$.

Embora a relação com mortalidade ainda não esteja clara, prolongar o tempo de permanência intra-hospitalar de idosos atrasando seu tratamento definitivo pode não só aumentar sua morbidade e predispor a infecções hospitalares como também encarecer os custos assistenciais( ${ }^{(21)}$.

Os dados referentes à taxa de infecção, reinternação e desfecho nos períodos estudados são expostos na tabela 5. Não houve diferença entre os grupos na taxa de infecção intra-hospitalar $(p=0,539)$. A análise 
descritiva encontrada em nosso estudo reflete o que também é relatado na literatura, onde cerca de $18 \%$ dos pacientes evoluem com infecção durante o internamento(24). Em relação ao foco infeccioso os mais comuns foram via aérea inferior $(13,6 \%$ no grupo 1 vs. $6 \%$ no grupo 2$)$ e infecções de trato urinário $(4,5 \%$ no grupo 1 vs. $5 \%$ no grupo 2), compatível com estudos semelhantes que descrevem a ocorrência principalmente de infecções respiratórias e do trato urinário. Entretanto somente uma porcentagem menor desses pacientes tende a evoluir de forma grave em decorrência de tais infecções, sendo a principal

Tabela 5 - Taxa de infecção, reinternação e desfecho pré e pós COMAFA, Curitiba/PR, Brasil, 2020

\begin{tabular}{lccc}
\hline & Pré COMAFA & Pós COMAFA & \\
Presença de infecção & $\mathbf{n}=\mathbf{8 8}$ & $\mathbf{n}=\mathbf{1 0 0}$ & $\mathbf{p}^{*}$ \\
\hline Não & $83 \%(\mathrm{n}=73)$ & $87 \%(\mathrm{n}=87)$ & 0,539 \\
Sim & $17 \%(\mathrm{n}=15)$ & $13 \%(\mathrm{n}=13)$ &
\end{tabular}

\begin{tabular}{lccc} 
Desfecho & & & $\mathbf{p}^{*}$ \\
\hline Alta & $84,1 \%(\mathrm{n}=74)$ & $93 \%(\mathrm{n}=93)$ & 0,065 \\
óbito & $15,9 \%(\mathrm{n}=14)$ & $7 \%(\mathrm{n}=7)$ &
\end{tabular}

\begin{tabular}{lccc} 
Necessidade de reinternação & & p $^{*}$ \\
\hline Não & $81,8 \%(\mathrm{n}=72)$ & $91 \%(\mathrm{n}=91)$ & 0,085 \\
$\operatorname{Sim}$ & $18,2 \%(\mathrm{n}=16)$ & $9 \%(\mathrm{n}=9)$ &
\end{tabular}

Fonte: os autores, 2020

*Variáveis analisadas através do teste exato de Fisher. Foi considerado como estatisticamente significante valores de $\mathrm{p}<0,05$.

manifestação de gravidade a insuficiência respiratória(24-26).

Em nosso estudo houve uma tendência estatística à redução da taxa de óbitos após a COMAFA $(p=0,065)$; a análise dos percentuais nos mostra que o número de óbitos após a implantação da equipe caiu mais da metade (15,9\% de óbitos antes vs. $7 \%$ após). Talvez o resultado encontrando na forma de tendência tenha sido fruto do pequeno número amostral de nosso estudo, e poderia vir a se tornar significativo caso fossem incluídos mais prontuários, já que os resultados obtidos em estudos semelhantes mostram que o cuidado multiprofissional para o idoso é mais eficaz, diminuindo a mortalidade e melhorando o prognóstico (OR 0,68; $p=0,03)^{(27)}$. Assim também o fez uma revisão sistemática recente, que demonstrou que a inclusão de cuidado multidisciplinar resultou em diminuição estatisticamente significativa no tempo até a cirurgia $(p=0,045)$, tempo de internamento $(p=0,0036)$ e taxas de mortalidade pósoperatória $(p=0,0034)^{(22)}$.

Em nosso estudo a análise de regressão logística binária mostrou redução na taxa de readmissão após a implantação da comissão multidisciplinar (OR $0,32 \quad 95 \%$ IC $\quad[0,11-0,84]$ $p=0,021$ ), independente do tempo de fratura até o tratamento, sexo e comorbidades (Tabela 2). Observa-se no geral que infecções tardias como pneumonias e septicemias são frequentemente relatadas como causas de readmissões pós-internação. Embora essas infecções possam ser decorrentes de alguma doença pré-existente, da vulnerabilidade relativa à idade e do impacto do trauma ou do procedimento cirúrgico que pode reduzir a reserva funcional do indivíduo, também podem estar associadas ao cuidado durante a internação e após a alta. Sendo assim, um percentual das readmissões por essas causas poderia ser evitado por meio de políticas relacionadas ao cuidado com o paciente ${ }^{(28)}$ 


\section{CONCLUSÃO}

A partir deste estudo, conclui-se que a implantação de uma comissão multidisciplinar propicia um tratamento médico mais rápido, diminuindo o tempo de hospitalização e a taxa de readmissão hospitalar em idosos com fratura de fêmur proximal. Além disso, evidencia-se uma importante redução da morbi-mortalidade intra-hospitalar desses pacientes.

Tais fatores são extremamente relevantes no contexto do envelhecimento populacional e aumento da prevalência de doenças crônicas. A busca de alternativas que otimize os cuidados de saúde desses pacientes, por meio de uma visão especializada e multidisciplinar, é um fator preponderante para a melhora dos indicadores hospitalares e a desoneração dos sistemas de saúde.

Tendo em vista os resultados apresentados, pode-se afirmar que o implemento de uma equipe multidisciplinar é de suma importância em serviços de traumatologia e pode ser utilizado como modelo de abordagem medico-hospitalar.

\section{CONFLITO DE INTERESSE}

Os autores do manuscrito intitulado "Avaliação da Implementação de Comissão Multidisciplinar para o Cuidado de Idosos com Fratura de Fêmur Proximal" declaram, para os devidos fins, que não há conflitos de interesses factuais ou potenciais, sejam eles de natureza financeira, pessoal, acadêmica, relacionado à afiliação institucional, política e/ou religiosa em relação ao artigo.

\section{REFERÊNCIAS}

1. American Academy of Orthopaedic Surgeons. Management of Hip Fractures in the Elderly: Evidence-Based Clinical Practice Guideline. 2014. Disponível em: https:// aaos.org/globalassets/quality-and-practiceresources/hip-fractures-in-the-elderly/ management_of_hip_fractures_in_the_elderly7-24-19.pdf.
2. Butt DA, Mamdani M, Austin PC, Tu K, Gomes T, Glazier RH. The Risk of Hip Fracture After Initiating Antihypertensive Drugs in the Elderly. Arch Intern Med. 2012;172(22):1739_ 1744. doi:10.1001/2013.jamainternmed.469

3. Craik J, Geleit R, Hiddema J, Bray E, Hampton R, Railton G, Ward D, Windley J. The effect of time to surgery on outcomes and complication rates following total hip arthroplasty for fractured neck of femur. Ann R Coll Surg Engl. 2019 May;101(5):342-345. doi: 10.1308/rcsann.2019.0032. Epub 2019 Mar 11. PMID: 30854861; PMCID: PMC6513369.

4. Cunha PTS, Artifon AN, Lima DP, Marques WV, Rahal MA, Ribeiro RR, Kitadai FT. Hip fractures in the elderly: surgical treatment timing and its correlation with delirium and infection. Acta Ortopédica Brasileira, 2008; 3(16):173-176. doi: https:// doi.org/10.1590/S1413-78522008000300010.

5. Berggren $M$, Stenvall $M$, Englund $U$, Olofsson B, Gustafson Y. Co-morbidities, complications and causes of death among people with femoral neck fracture - a three-year follow-up study. BMC Geriatr. 2016 Jun 3;16:120. doi: 10.1186/s12877-016-0291-5. PMID: 27260196; PMCID: PMC4893237.

6. Dhanwal DK, Dennison EM, Harvey NC, Cooper C. Epidemiology of hip fracture: Worldwide geographic variation. Indian J Orthop. 2011 Jan;45(1):15-22. doi: 10.4103/0019-5413.73656. PMID: 21221218; PMCID: PMC3004072.

7. Arliani GG, Costa D, Linhares, AGK, Balbachevsky $\mathrm{H}$, Fernandes JA, Reis, FB. Correlation between time until surgical treatment and mortality among elderly patients with fractures at the proximal end of the fêmur. Revista Brasileira de Ortopedia. 2011; 2(46) 189-194. DOI: https://doi.org/10.1590/S010236162011000200013.

8. Avelino NR, Chaoubah A, Fernandes GC, Loures FB, Ortega GP, Magacho FLR, Gonçalves PJ. Análise do custo-efetividade da 
implantação de uma Unidade de Prática Integrada no cuidado de idosos com fratura de quadril. J. bras. econ. Saúde. 2020; 12(2) 121127. Disponível em: https://repositorio.ufjf.br/ jspui/handle/ufjf/8622?locale=pt_BR.

9. Farias FID, Terra NL, Guerra MTE. Evaluation of the effectiveness of a care program for elderly patients with hip fractures: a network strategy. Revista Brasileira de Geriatria e Gerontologia. 2017; 20(5) 702-712. doi: https://doi.org/10.1590/1981-

22562017020.170008

10. Dyer SM, Crotty $M$, Fairhall $N$, Magaziner J, Beaupre LA, Cameron ID, Sherrington C; Fragility Fracture Network (FFN) Rehabilitation Research Special Interest Group. A critical review of the long-term disability outcomes following hip fracture. BMC Geriatr. 2016 Sep 2;16(1):158. doi: 10.1186/s12877016-0332-0. PMID: 27590604; PMCID: PMC5010762.

11. Porter ME, Lee TH. A estratégia que irá corrigir os serviços de saúde. Harvard Business Review. 2013; 32-49.

12. Farias FID, Terra NL, Brum RL, Alves F, Frare CS, Guerra MTE. Determining factors in the costs of treatments for elderly people with hip fracture. Geriatr Gerontol Aging. 2016;10:196-202.

13. Rodriguez-Fernandez $P$, AdarragaCansino D, Carpintero P. Effects of delayed hip fracture surgery on mortality and morbidity in elderly patients. Clin Orthop Relat Res. 2011 Nov;469(11):3218-21. doi: 10.1007/s11999010-1756-z. Epub 2011 Jan 6. PMID: 21210312; PMCID: PMC3183186.

14. Graphpad Prism versão 7.00 para Windows, GraphPad Software, La Jolla California USA, www.graphpad.com.

15. Zar, J. H. Biostatistical Analysis. 5ed, 950p. Prentice Hall. 2009.

16. Givens JL, Sanft TB, Marcantonio ER.
Functional recovery after hip fracture: the combined effects of depressive symptoms, cognitive impairment, and delirium. J Am Geriatr Soc. 2008 Jun;56(6):1075-9. doi: 10.1111/j.1532-5415.2008.01711.x. Epub 2008 Apr 18. PMID: 18422945.

17. Leibson CL, Tosteson AN, Gabriel SE, Ransom JE, Melton LJ. Mortality, disability, and nursing home use for persons with and without hip fracture: a population-based study. J Am Geriatr Soc. 2002 Oct;50(10):1644-50. doi: 10.1046/j.1532-5415.2002.50455.x. PMID: 12366617.

18. Vidal EI, Coeli CM, Pinheiro RS, Camargo KR Jr. Mortality within 1 year after hip fracture surgical repair in the elderly according to postoperative period: a probabilistic record linkage study in Brazil. Osteoporos Int. 2006 Oct;17(10):1569-76. doi: 10.1007/s00198-0060173-3. Epub 2006 Jul 27. PMID: 16871434.

19. Roche JJ, Wenn RT, Sahota O, Moran CG. Effect of comorbidities and postoperative complications on mortality after hip fracture in elderly people: prospective observational cohort study. BMJ. 2005 Dec 10;331(7529):1374. doi: 10.1136/ bmj.38643.663843.55. Epub 2005 Nov 18. PMID: 16299013; PMCID: PMC1309645.

20. Moja L, Piatti A, Pecoraro V, Ricci C, Virgili G, Salanti G, Germagnoli L, Liberati A, Banfi $G$. Timing matters in hip fracture surgery: patients operated within 48 hours have better outcomes. A meta-analysis and metaregression of over 190,000 patients. PLoS One. 2012;7(10):e46175. doi: 10.1371/ journal.pone.0046175. Epub 2012 Oct 3. PMID: 23056256; PMCID: PMC3463569.

21. Khan SK, Kalra S, Khanna A, Thiruvengada MM, Parker MJ. Timing of surgery for hip fractures: a systematic review of 52 published studies involving 291,413 patients. Injury. 2009 Jul;40(7):692-7. doi: 10.1016/j.injury.2009.01.010. Epub 2009 May 18. PMID: 19450802. 
22. Patel JN, Klein DS, Sreekumar S, Liporace FA, Yoon RS. Outcomes in Multidisciplinary Team-based Approach in Geriatric Hip Fracture Care: A Systematic Review. J Am Acad Orthop Surg. 2020 Feb 1;28(3):128-133. doi: 10.5435/JAAOS-D-1800425. PMID: 31977613.

23. Wallace R, Angus LDG, Munnangi S, Shukry S, DiGiacomo JC, Ruotolo C. Improved outcomes following implementation of a multidisciplinary care pathway for elderly hip fractures. Aging Clin Exp Res. 2019 Feb;31(2):273-278. doi: 10.1007/s40520-0180952-7. Epub 2018 Apr 23. PMID: 29687304.

24. Uliana CS, Abagge M, Malafaia O, Filho FAK, Cunha LAM. Fraturas transtrocantéricas Avaliação dos dados da admissão à alta hospitalar. Revista Brasileira de Ortopedia. 2014; 49(2):121-128. doi: https://doi.org/ 10.1016/j.rbo.2014.01.006.

25. Edelmuth SVC, Sorio GN, Sprovieri AA, Gali JC, Peron SF. Comorbidities, clinical intercurrences, and factors associated with mortality in elderly patients admitted for a hip fracture. Revista Brasileira de Ortopedia. 2018; 53(5):543-551. doi: https://doi.org/10.1016/ j.rboe.2018.07.014.

26. Rostagnoi C, Mannucci M, Cartei A, Polidori GL, Buzz R, Landi F, Gensini GF. Multidisciplinary Approach to Hip Fracture in the Elderly: Florence Experience. Emergency Medicine. 2013; 4(3). doi: https://doi.org/ 10.4172/2165-7548.1000148.

27. Middleton M, Wan B, da Assunçao R. Improving hip fracture outcomes with integrated orthogeriatric care: a comparison between two accepted orthogeriatric models. Age Ageing. 2017 May 1;46(3):465-470. doi: 10.1093/ageing/ afw232. PMID: 27974304.

28. Paula FL, Cunha GM, Leite IC, Pinheiro RS, Valente JG. Elderly readmission and death after discharge from treatment of hip fracture, occurred in public hospitals from 2008 to 2010 , Rio de Janeiro. 2015; 18(2):439-453. doi: https://doi.org/10.1590/1980-

5497201500020012. 